\title{
A Case Based Study for Urban Traffic Control
}

\author{
Abdul Mateen ${ }^{1}$ Fahim Arif $^{2}$ Shahid Kamal ${ }^{3}$ \\ ${ }^{1}$ Department of Computer Science, Federal Urdu University of Arts, Science \& \\ Technology, Islamabad, Pakistan \\ ${ }^{2}$ Computer Science Department, Military College of Signals, National \\ University of Sciences \& Technology, Islamabad, Pakistan \\ ${ }^{3}$ Faculty of Computer and Information Systems, University Technology Malaysia, \\ Skudai, Johor Bahru, Malaysia
}

\begin{abstract}
Traffic management especially in big cities is a common problem in all over the world. Therefore, traffic management problem on the signals is getting importance and should be solved to save the fuel, time and environment as well. The proposed research is based on the traffic flow and assigns time accordingly. It also provides a way for safe and fast passage for emergency vehicles by sending their information to next signals. The proposed traffic management solution is followed by a case study that reveals the performance and effectiveness of the solution.
\end{abstract}

Keywords: Traffic, Intelligent, Controller

\section{Introduction}

Number of vehicles that passes to the traffic signal at some specific time is known as traffic. Main entities of the traffic control include roads, vehicles and signals. The problem of the traffic congestion happens due to the larger traffic demand than the available vehicle passage or unsuitable time interval to each side of the signal. Traffic jam also occurs due to the many other interrelated real world problems such as more traffic flow than the availability, wrong lane turns, sudden and unauthorized pedestrian crossing, speed lane violations, police check posts, operation of the emergency services etc.

History of traffic signal starts from December, 1868 where traffic is controlled at the London by J. P. Knight. The traffic signal at that time has red and green lights. There was a lever to switch the lights from red to green and vice versa. In 1912 modern traffic signals with red and green electric traffic signals [1] were introduced by Lester Wire. Later on, in 1914 an American traffic signal company put the traffic signals at the Ohio State that was consist of red and green lights with a buzzer. The 4-way traffic signal was introduced by the Willium Pots in 1920. First interconnected traffic signal was introduced in 1917. This signal was installed at the Salt Lake City, USA with six junctions and operated through human being (manually).

Traffic control can be performed through 3 methods (Fixed, Actuated and adoptive). One way is to assign a fixed interval by considering previous data. To overcome the cons of Pre-time traffic signal, actuated control method is adopted where the push buttons (detectors) are used. They provide information to actuator and assign time according to the traffic flow. Adaptive traffic control has the capability to assign time according to the 
current traffic flow and continuously learns from its past.

Rest of the paper is organized as follows. Section 2 describes the previous work that has been done in the context of traffic control and management. Section 3 presents a case study that is based on the traffic data of Islamabad, capital of Pakistan. Section 4 portrays the proposed solution for the traffic control and management while section 5 concludes the paper with future work.

\section{Related Work}

A lot of research related with traffic control [3-13] is available in the form of research papers, white paper, technical reports and case studies. Some of these which are closely related discussed here.

A model for an autonomous traffic control system is introduced by Alagar et al. [3]. A decentralized approach is adopted to manage the traffic at signals. The Vehicle Routing Problems (VRP) is elaborated by Sam et al. [4] and solution for the VRP is provided through agent oriented architecture. Lee et al. [7] proposed a Traffic Parameter Measure Algorithm to derive traffic parameters (vehicle quantity, speed etc) from the video image sequences. A multi-agent architecture is adopted to design an Intelligent Transport System by Liu et al. [9] that give traffic related information to drivers that assist them to make correct decisions. Albagul et al. [11] simulated the intelligent traffic signal which detects vehicles and provides the green signal time accordingly. Yi-Sheng Huang [12] provided a model for the traffic light control using the state chart diagram which provides the visual formalism of the system. Shamshirband et al. [13] introduced a multi-agent and Weighted Strategy Sharing (WSS) technique for cooperative learning in traffic management system.
Main problem with human administrated traffic management system is poor response time while in automatic traffic management system allocation of constant time to each side creates traffic congestion, wastage of time and energy. Other problem with both systems is lack of entertaining the rescue or emergency vehicles while the proposed solution will detect the rescue vehicles and send this information to the next signals in advance for safe and immediate passage.

\section{Case Study}

Islamabad, capital of Pakistan is facing the traffic congestion situation and still implementing the static traffic management architecture which causes wastage of time and energy. Serious attention is required to resolve this issue by adopting intelligent and autonomous traffic management solution. In case of independent traffic signal, following formulae are used to calculate the different parameters for the allocation of signal time:

$$
\text { Assigned Time }=\text { Exit Time }\left(\text { for } 1^{\text {st }} \text { row }\right)+
$$

$$
0.72 \text { (Rows - 1) }
$$

Exit time $\left(1^{\text {st }}\right.$ row $)=3600 / 10000 *$ Distance Exit time (next rows) $=(3600 / 10000 * 2)$

$$
\begin{aligned}
& *(\text { Rows }-1) \\
= & (0.72(\text { Rows }-1)
\end{aligned}
$$

No. of Rows $=$ Round (vehicles/Lanes)

Where Exit Time is Time to cross signal Distance: Signal Width

\section{If (Assigned Time > Threshold) Then Assigned Time $=$ Threshold}

Suppose threshold for signal is $50 \mathrm{secs}$ and numbers of vehicles on each side are 20, 9, 4 and 17. These are represented through variables V1, V2, V3 and V4 respectively. Here, road has 2 lanes with 10 meter of distance. If assigned time for one side of signal is $<60$ seconds then 
calculated time is used otherwise Threshold (60) will be used as a signal time.

$$
\begin{aligned}
& +0.72 *(\text { Rows- } 1)) \\
& T 1=3.6+0.72(9) \\
& =10.08 \mathrm{sec} \\
& \text { If }(10.08>60) \text { then } \\
& T 1=60
\end{aligned}
$$

In this case above condition is false, therefore $\mathrm{T} 1$ will remain $10.08 \mathrm{sec}$.

\section{Signal 2}

$T 2=$ Exit Time $\left(\right.$ for $1^{\text {st }}$ row $)+0.72$ (4)

$T 2=3.6+2.88$

$=6.48 \mathrm{sec}$

\section{Signal 3}

$$
\begin{aligned}
\text { T3 } & =\text { Exit Time }\left(\text { for } 1^{\text {st }} \text { row }\right)+0.72(3) \\
& =3.6+2.16 \\
& =5.76 \mathrm{sec}
\end{aligned}
$$

\section{Signal 4}

$$
\begin{aligned}
T 4 & =\text { Exit Time }\left(\text { for } 1^{\text {st }} \text { row }\right)+0.72(8) \\
& =3.6+5.76 \\
& =9.36 \mathrm{sec}
\end{aligned}
$$

Signal Time $=T 1+T 2+T 3+T 4+$ Alert Time $=(10.08+6.48+5.76+9.36)+20$ $=51.68 \mathrm{sec}$

Where 20 secs is signal alert time that has same effect on static traffic management architecture and proposed solution. Order of signal opening will be 3,2, 4 and 1 . When allocated time of signal 3 is completed, DM agent will show the yellow light $(5 \mathrm{sec})$ and then red and finally open the next signal (signal 2) in above case and so on. The average waiting time for above example will be calculated as: Signal $3 A W T=0$

Signal $2 A W T=T 3 / V 2=0.64$

Signal $4 A W T=(T 3+T 2) / V 4=0.72$

Signall AWT $=(T 2+T 3+T 4) / V 1=1.08$ $A W T($ for All Vehicles $)=2.44$

\section{Proposed Solution}

Traffic management problem can easily be tackled with the use of software agents $[2,6]$. There will be an agent for each component in the proposed solution. All the agents are autonomous and have capability to take decision or any action according to the traffic volume. The proposed system will consist of mainly three main components, Environmental, Observer and the knowledge base. Environmental agent will consist of camera and sound sensor, Knowledge base is the data repository while the observer agent consists of three sub-agents, i.e. Analyzer agent, Decision-Making agent, and the Learner agent as shown in Fig. 1.

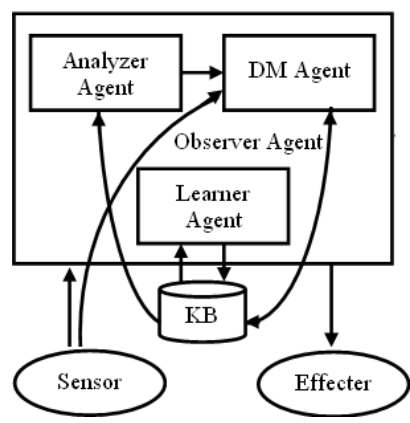

Fig. 1: Components of Proposed Traffic Management Solution.

The proposed solution for traffic management will take the information about current traffic via sensors that may be either camera or sonic sensor [10]. The collected information about the traffic will be passed to the Analyzer agent where vehicles are counted on each side of traffic signal. The vehicle count on each side of the signal will be sent to the DM agent. On the basis of traffic volume on each side, suitable time interval will be assigned. The DM agent will analyze the traffic volume on each side and allocates threshold for a signal which will also be updated after each an hour. These steps 
will be repeated after the completion of signal threshold. In case of rescue alert (from the sonic sensor), DM agent will store the current state of signals into the Knowledge Base, close the opened traffic signal and open the signal from where the emergency vehicle is approaching. When rescue vehicle will be passed, the previous state of the signals and opening sequence will be retrieved from the KB. After restoring the previous sequence, information about the rescue vehicle will be communicated to the next signal for its safe and immediate passage.

\section{Conclusion}

An autonomous traffic control solution for urban traffic has been proposed that controls the traffic on traffic signals with minimum human involvement. Furthermore, it will be featured by the special treatment for the rescue and emergency vehicles. The benefits of proposed system include less human effort, dynamic decisions, maximum traffic flow and less fuel consumption.

\section{References}

[1] E. A. Mueller, "Aspects of the History of Traffic Signals", IEEE Trans on Vehicular Technology, volume 19 (1), pp. 6-17, 1970.

[2] S. Alagar, and D. Muthiayen, "A Rigorous Approach to Modeling Autonomous Traffic Control Systems", $6^{\text {th }}$ International Symposium on Autonomous Decentralized Systems, pp. 193-200, 2003.

[3] R. Sam, T. Olena, and W. Mennell, "An Agent Architecture for Vehicle Routing Problems", SAC ACM, 2001.

[4] G. Pour, "Expanding the Possibilities for Enterprise Computing: MultiAgent Autonomic Computing", $10^{\text {th }}$
IEEE International EDOCW, pp. $33-$ 33, 2006.

[5] Y. Lee, and Y. T. Lee, "A Fast Algorithm for Measuring Traffic Vehicle Parameters", Proceedings of the $7^{\text {th }}$ International Conference on Machine Learning \& Cybernetics, pp. 3061-66, 2008.

[6] N. Kasun, and Y. Janaka, "Optimization of Traffic Signal Light Timing Using Simulation", Winter Simulation Conference, pp. 1428-1433, 2004.

[7] L. Xiaonan, and F. Zhiyi, "An AgentBased Intelligent Transport System", $11^{\text {th }}$ International Conference on Computer Supported Cooperative Work in Design (CSCWD), pp. 304315, 2007.

[8] C. Michael, "MPEG-7 Sound Recognition Tools", Mitsubishi Electric Research Labs, Cambridge, MA, Unites States of America.

[9] A. Albagul, M. Hrairi, Wahyudi and M. F. Hidayathullah, "Design and Development of Sensor Based Traffic Light System", American Journal of Applied Sciences 3 (3), 2006, pp. 1745-1749.

[10] H. Yi-Sheng, "Design of Traffic Light Control Systems Using State charts", The Computer Journal, 49(6), 2006.

[11] S. S. Shamshirband, H. Shirgahi, M. Gholami, and B. Kia, "Coordination between Traffic Signals Based on Cooperative", World Applied Sciences Journal, 5 (5), pp. 525-530, 2008.

[12] R. Stuart, and N. Peter, "Artificial Intelligence, A Modern Approach", $2^{\text {nd }}$ Edition, ISBN No. 81-7758-367-0, 2008.

[13] J. Tian, and H. Tianfield, "A MultiAgent Approach to the Design of an E-medicine System”, Springer-Verlag Berlin Heidelberg, 2003. 